\title{
A Rapid Penetration Trajectory Optimization Method for Hypersonic Vehicles
}

\author{
Binbin Yan, Ruifan Liu (D, Pei Dai, Muzeng Xing, and Shuangxi Liu \\ School of Astronautics, Northwestern Polytechnical University, Xi'an 710072, China \\ Correspondence should be addressed to Ruifan Liu; liuruifan@mail.nwpu.edu.cn
}

Received 8 January 2019; Revised 13 April 2019; Accepted 13 June 2019; Published 9 July 2019

Academic Editor: Maj D. Mirmirani

Copyright (C) 2019 Binbin Yan et al. This is an open access article distributed under the Creative Commons Attribution License, which permits unrestricted use, distribution, and reproduction in any medium, provided the original work is properly cited.

\begin{abstract}
The evasion maneuver problem of hypersonic vehicles differs from those of ballistic missiles and other traditional weapons, showing distinctive properties including expansive maneuver range and weak maneuverability. How to avoid the disadvantage of low available overload and ensure follow-up tasks are the main concerns of the hypersonic penetration. This paper presents a penetration trajectory optimization algorithm for an air-breathing hypersonic vehicle, where the prerequisite penetration condition is analyzed and control costs are chosen as an objective function to minimize the fuel consumption and maneuver range. This paper focuses on how to formulate the complex, highly constrained nonconvex penetration problem to be a sequence of easily solved second-order cone programming through a combination of successive linearization and relaxation techniques. Innovation lies in the raising of the penetration angle and the relaxation technique of nonlinear and nonconvex elements. Various numerical simulations are conducted to verify the validity of penetration condition and to demonstrate that the proposed method is effective and has a good computational performance irrespective of initial guesses.
\end{abstract}

\section{Introduction}

Since the hypersonic vehicles have arrived, the attack and defense countermeasure game centered on it receives the focal attention by various military powers. The air-breathing hypersonic vehicle cruises at the altitude of the near space, featuring a lasting and wide-range maneuverability. But owing to the usage of the unique integrated propulsion technology and the scramjet engine [1], strict constraints are also imposed on its maneuverability, including the attack angle and bank angle $[2,3]$. Therefore, the hypersonic penetration possesses characteristics of a "wide maneuvering range, weak maneuverability, and high flight speed." Besides, the prominent infrared features of hypersonic vehicles make it easily to be detected and intercepted. Consequently, how to make maximal use of the speed superiority and achieve penetration with minimal energy consumption are the core of hypersonic penetration programming.
In the confrontation, the hypersonic vehicle and the interception missile maneuver actively and simultaneously, thus always regarded as a complicated gaming problem. With the game theory, differential gaming models are established. Shaferman and Shima $[4,5]$ studied the zero-sum differential game theory, the quadratic differential game theory, and various other forms of differential game and analyzed the conditions in which the saddle points of the differential game exist. However, most of studies of differential game theory focus on mathematical modeling and theoretical derivation, while its application to practical engineering remains a challenge [6] . Considering the huge speed gap, the game problem between a hypersonic vehicle and an interception missile is not like the normal one. In other words, only when harsh interception conditions are satisfied can the interceptor succeed. Accordingly, Dwivedi et al. [7] presented a midcourse guidance law against high-speed targets believing that alignment angle constraints in both elevation and azimuth could create 
a favorable condition for the terminal guidance while Guo et al. [8] tried to guide a hypersonic vehicle to break the alignment angle of the interceptor from an opponent view; thus, they converted the penetration problem into an optimal control problem.

In fact, using optimal control techniques for guidance of flight vehicles is not new. Numerical methods, like the shooting method [9], the gradient method [10], and the recently prominent one, the pseudospectral method [11, 12], have been developed to solve two-point boundary value problems (TPBVPs) derived from optimal problems. However, all of them lead to large computations that are infeasible to implement in real time. Other contributions have also been proposed in this regard, such as the model predictive control (MPC) theory [13] combined with approximate dynamic programming (APC) and model predictive static programming (MPSP) [7, 8]. The former one has been successfully used in the industry (especially in slow-varying process control) but is not guaranteed to remain optimal and stable for its sliding time window $[13,14]$. As to MPSP which is rather efficient and adaptable, however, when applied to the hypersonic vehicle, it is also not a preferable method since it cannot properly solve the optimal problems with control constraints.

This paper is aimed at designing a rapid penetration method to generate an appropriate evasion trajectory of a hypersonic vehicle against a head-on intercepting missile. According to the above content, the main two barriers of this problem are an explicit expression of penetration condition and a rapid, robust optimization algorithm catering both terminal angular constraints and the demanding control constraints of hypersonic vehicles. About the former, this paper introduces the concept of the penetration angle on the basis of interception geometry, giving the clear formula of penetration condition. The validity of the condition is also verified by the numerical simulation in terms of the ultimate miss distance under different penetration angles. As to the feasible optimization method, this paper raises a method based on sequential convex programming, which could meet the control constraints as well as the penetration angle constraints in a short time, and compared with the existing optimal method GPM, the algorithm proposed in this paper has a very competitive convergence rate and does not require good initial guesses.

Convex optimization has been widely utilized in recent years for its unique theoretical advantages and the polynomial time complexity $[15,16]$. However, applications of convex optimization in aerospace trajectory planning have been limited to problems without complex nonlinear dynamics and aerodynamic forces so far. This paper is mainly devoted to this difficulty in the penetration application. The technique developed in this paper successfully "convexifies" the initial nonconvex penetration problem into a series of second-order cone programming (SOCP) problems. Various convexity strategies are applied to different nonconvex elements. The SOCP-based method proves to be a very robust and rapid tool for hypersonic penetration trajectory optimization. Numerical results are provided to demonstrate the effectiveness and some of the noteworthy aspects of the method.

The rest of this paper is organized as follows. In Section 2, the countermeasure model is established, including the hypersonic vehicle model, the interceptor model, and the relative motion model, and thus, the original form of penetration problem is described. In Section 3, the convexification process is explained in detail and the converted SOCP problem is presented. Numerical simulation results are presented in Section 4. Finally, some conclusions are drawn in Section 5 .

\section{Mathematical Model for Attack and Defense Countermeasure}

The primary objective of this paper is to present an effective penetration guidance logic. Hence, considering solving speed and application feasibility, one only requires point mass mathematical models for both the vehicle and the interception missile. Moreover, with the complex high-fidelity model, the calculation of Jacobi matrices presented in Section 3 will be boosted. Therefore, it is crucial to formulate the penetration problem in a certain simplified way conducive to the SOCP approach. This section presents the details of one such formulation of the problem.

2.1. Dynamics of the Hypersonic Vehicle. The penetration maneuver of a hypersonic vehicle is a short-range trajectory optimization problem; for this reason, we simplify its kinematic equation sets as follows:

(a) With the rotation of the Earth not taken into account, namely, ignoring the Coriolis acceleration and convective acceleration

(b) Assuming that the Earth is flat, namely, ignoring the influence of its centrifugal force

And as is mentioned above, this paper pays most of its attention to the trajectory programming; therefore, the mass point model is established and some simplifications of the vehicle's thrust and aerodynamic forces are conducted.

Thus, we obtain the following equations of the mass point motion model of the hypersonic vehicle:

$$
\left\{\begin{array}{l}
\dot{h}_{e}=V_{e} \sin \theta_{e}, \\
\dot{V}_{e}=\frac{T \cos \alpha_{T}-D}{m}-g \sin \theta_{e}, \\
\dot{\theta}_{e}=\frac{T \sin \alpha_{T} \cos \sigma+L_{T} \cos \sigma}{m V_{e}}-\frac{g \cos \theta_{e}}{V_{e}}, \\
\dot{\psi}_{e}=\frac{T \sin \alpha_{T} \sin \sigma+L_{T} \sin \sigma}{m V_{e} \cos \theta_{e}}, \\
\dot{m}_{e}=-m_{s},
\end{array}\right.
$$

where $h_{e}$ is the altitude of the hypersonic vehicle; $V_{e}$ is the speed; $\theta_{e}$ and $\psi_{e}$ represent the flight path angle and trajectory deflection angle; $\alpha_{T}$ and $\sigma$ are the angle of attack and bank 


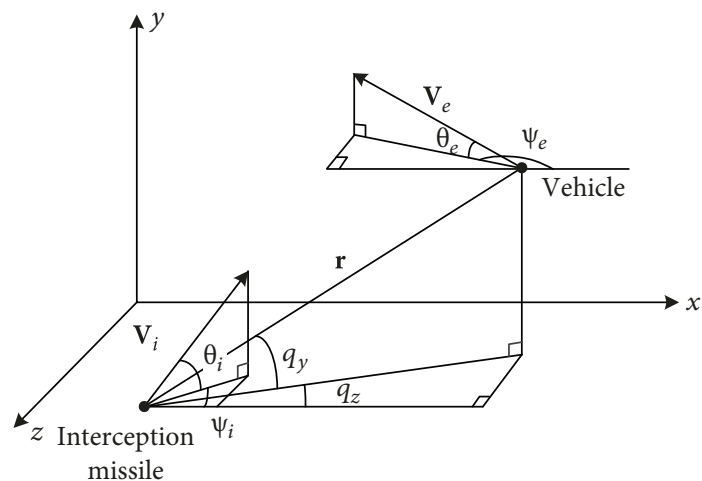

FIGURE 1: Relative kinetics in the attack and defense countermeasure.

angle; $L_{T}, D$, and $T$ are its aerodynamic force and thrust; $m_{e}$ is its mass; $m_{s}$ is its mass consumption rate.

The thrust model, the aerodynamic force model, and the control constraints are as follows:

$$
\begin{aligned}
& \left\{\begin{array}{l}
L_{T}=0.5 \rho V_{e}^{2} S\left(C_{L 0}+C_{L}^{\alpha} \alpha_{T}\right), \\
D=0.5 \rho V_{e}^{2} S\left(C_{D 0}+C_{D}^{\alpha^{2}} \alpha_{T}^{2}\right), \\
T=0.5 \rho V_{e}^{2} S C_{T}^{m_{s}} m_{s},
\end{array}\right. \\
& \left\{\begin{array}{l}
\alpha_{T \min } \leq \alpha_{T} \leq \alpha_{T \max }, \\
|\dot{\sigma}| \leq \dot{\sigma}_{\max }, \\
0 \leq m_{s} \leq m_{s \max },
\end{array}\right.
\end{aligned}
$$

where $\alpha_{T \text { min }}$ and $\alpha_{T \text { max }}$ represent the boundaries of attack of the angle while $\dot{\sigma}_{\max }$ and $m_{s \max }$ represent the upper limits of the bank angle rate and mass consumption rate, respectively.

2.2. Dynamics of the Interception Missile. For the midcourse guidance of an interception missile against a high-speed target, it is desirable to shape the missile trajectory that provides a preferable initial state for terminal attack. Instead of the guidance laws carried out according to the nonlinear theory and the MPSP algorithm, whose practicability are always restricted in application, this paper utilizes a more compendious and feasible guidance law called the trajectory shaping guidance law $[17,18]$.

The trajectory shaping guidance law has been developed to travel to the desired trajectory and verified by physical applications, such as landing the Apollo spacecraft on the moon. The guidance law appears to be a form of augmented proportional navigation plus an extra term that is proportional to the difference between the line of sight angle and the desired angle at the end of flight. The application formula for this paper is as follows:

$$
\left\{\begin{array}{l}
\dot{\theta}_{i}=N_{1} \dot{q}_{y}+N_{2}\left(t_{g o}\right)\left(q_{y}+\theta_{e}\right), \\
\dot{\psi}_{i}=N_{1} \dot{q}_{z}+N_{3}\left(t_{g o}\right)\left(q_{z}-\psi_{e}+\pi\right),
\end{array}\right.
$$

where $\theta_{i}$ and $\psi_{i}$ are the flight path angle and the trajectory deflection angle; $q_{y}$ and $q_{z}$ are the line of sight (LOS) angle in elevation and azimuth, respectively. Figure 1 shows explicit geometric meaning; $N_{1}, N_{2}, N_{3}$ are the constants of the guidance law, where $t_{g o}$ is the remaining flight time.

2.3. Attack and Defense Countermeasure Analysis. Under usual circumstances, the speed of a hypersonic vehicle is much higher than that of a defense system. Hence, only the head-on strike by an interception missile can impose a threat to the hypersonic vehicle. Through reasonable assumptions and simplifications, the interception geometry is established, and thus, one can gain the interception missile's attack area. The attack area is a round spherical surface that includes the interception missile but excludes the hypersonic vehicle, and its boundary is tangent to the hypersonic vehicle, as shown in Figure 2. See reference [19] for specific derivation processes.

It is known from interception geometry that if the velocity vector of the hypersonic vehicle is always outside of the interception geometric tangent line, namely, $\eta>\eta_{\max }$, then it can break through the head-on strike situation of the interception missile, achieving a successful penetration [20].

$\eta$ is defined as the penetration angle of the hypersonic vehicle, namely, the angle between the line-of-sight and the velocity of the hypersonic vehicle. Its cosine value can be obtained with the transformation matrices of the groundtrajectory coordinate system and the ground-LOS coordinate system. The relationship can be formulated as follows:

$$
\cos \eta=-\cos q_{y} \cos \theta_{e} \cos \left(q_{z}-\psi_{e}\right)-\sin \theta_{e} \sin q_{y} .
$$

2.4. Mathematical Model of Relative Motions. With the penetration angle constraints taken into consideration, the model of relative motion between the hypersonic vehicle and the interception missile is established under the LOS coordinate system. The spatial coordinates and relative kinetics in the attack and defense countermeasure are shown in Figure 1 .

The relative motions are defined by the following equations:

$$
\left\{\begin{array}{l}
\dot{r}=V_{e}\left[\cos \theta_{e} \cos q_{y} \cos \left(\psi_{e}-q_{z}\right)+\sin \theta_{e} \sin q_{y}\right]-V_{i}\left[\cos \theta_{i} \cos q_{y} \cos \left(\psi_{i}-q_{z}\right)+\sin \theta_{i} \sin q_{y}\right] \\
\dot{q}_{y} r=-V_{e}\left[\cos \theta_{e} \sin q_{y} \cos \left(\psi_{e}-q_{z}\right)-\sin \theta_{e} \cos q_{y}\right]+V_{i}\left[\cos \theta_{i} \sin q_{y} \cos \left(\psi_{i}-q_{z}\right)-\sin \theta_{i} \cos q_{y}\right] \\
\dot{q}_{z} r \cos q_{y}=V_{e} \cos \theta_{e} \sin \left(\psi_{e}-q_{z}\right)-V_{i} \cos \theta_{i} \sin \left(\psi_{i}-q_{z}\right)
\end{array}\right.
$$




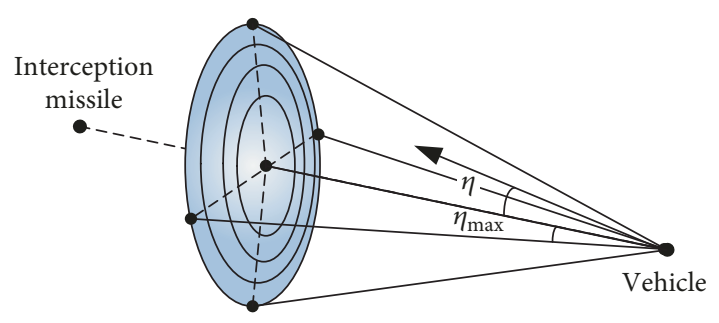

FIGURE 2: The three-dimensional interception attack area.

where $\left(r, q_{y}, q_{z}\right)$ are the relative distance, the LOS elevation angle, and the LOS azimuth angle between the hypersonic vehicle and the interception missile.

2.5. Hypersonic Vehicle's Penetration Problem. Taking the minimum control as the objective function and the penetration angle constraint into consideration, the hypersonic vehicle's penetration problem can be described as an optimal control problem $P_{0}$ :

$$
\begin{aligned}
& P_{0}: \min J=\int \mathbf{u}(t)^{T} R \mathbf{u}(t), \\
& \text { s.t. }\left\{\begin{array}{l}
\dot{\mathbf{x}}(t)=f(\mathbf{x}(t), \mathbf{u}(t)), \quad \mathbf{x}\left(t_{0}\right)=\mathbf{x}_{0}, \\
\alpha_{T \min } \leq \alpha_{T}(t) \leq \alpha_{T \max }, \\
|\dot{\sigma}(t)| \leq \dot{\sigma}_{\max }, \\
0 \leq m_{s}(t) \leq m_{s \max }, \\
\eta(t)>\eta_{\min },
\end{array}\right.
\end{aligned}
$$

where the control vector is $\mathbf{u}=\left[\alpha_{T}, \sigma, m_{s}\right]^{T} \in \mathbf{R}^{3} ; \mathbf{x}=\left[h_{e}, V_{e}\right.$, $\left.\theta_{e}, \psi_{e}, m_{e}, \theta_{i}, \psi_{i}, r, q_{y}, q_{z}\right]^{T} \in \mathbf{R}^{11}$ represents the state vector.

\section{Penetration Problem Convexification}

The hypersonic penetration problem $P_{0}$ has nonlinear inequation constraints and other nonconvex factors brought about by the quadratic integral objective function, nonlinear differential equation constraints, and penetration angle constraints. Therefore, how to transform the penetration problem into a convex problem that can be easily solved is crucial for applying a convex optimization algorithm. The following section will explain in some detail the processes.

3.1. Linearization and Discretization of Dynamics Equations. The nonlinear differential equations are approximated by a series of linear time-varying systems, and the solution sequence of linear systems, through several iterations, will be convergent to the optimal solution of the original system [21].

For simplicity of notation, the nonlinear state equation is expressed as follows:

$$
\dot{\mathbf{x}}=f(\mathbf{x}, \mathbf{u}), \quad \mathbf{x}\left(t_{0}\right)=\mathbf{x}_{0} .
$$

In the form of state correlation coefficient, the nonlinear state equations can be presented as follows:

$$
\left\{\begin{array}{l}
\dot{\mathbf{x}}=F_{x}(\mathbf{x}, \mathbf{u}) \mathbf{x}+F_{u}(\mathbf{x}, \mathbf{u}) \mathbf{u}+b(\mathbf{x}, \mathbf{u}), \\
b(\mathbf{x}, \mathbf{u})=f(\mathbf{x}, \mathbf{u})-F_{x}(\mathbf{x}, \mathbf{u}) \mathbf{x}-F_{u}(\mathbf{x}, \mathbf{u}) \mathbf{u},
\end{array}\right.
$$

where $F_{x}=\partial \mathbf{f} / \partial \mathbf{x}$ and $F_{u}=\partial \mathbf{f} / \partial \mathbf{u}$ are the Jacobi matrices of the state function $\mathbf{f}$ in terms of $\mathbf{x}$ and $\mathbf{u}$. If the above equations meet the local Lipschitz condition, then the solution of the nonlinear state equation can be approximated with the following linear time-varying equation:

$$
\begin{aligned}
\dot{\mathbf{x}}^{k+1}= & F_{x}\left(\mathbf{x}^{k}, \mathbf{u}^{k}\right) \mathbf{x}^{k+1}+F_{u}\left(\mathbf{x}^{k}, \mathbf{u}^{k}\right) \mathbf{u}^{k+1} \\
& +b\left(\mathbf{x}^{k}, \mathbf{u}^{k}\right), \quad k=0,1, \cdots
\end{aligned}
$$

where $\mathbf{x}^{k}$ and $\mathbf{u}^{k}$ denote the values of the state variable and control variable in the $k$ times of iteration.

The sequences of solutions obtained through solving the above linear time-varying equations converge to the solutions of original nonlinear equations [22], namely,

$$
\lim _{k \rightarrow \infty}\left\{\mathbf{x}^{k}(t)\right\} \rightarrow \mathbf{x}(t) .
$$

To ensure the effectiveness of state equation linearization, we add thrust domain constraints to state variables.

$$
\left\|\mathbf{x}-\mathbf{x}^{k}\right\| \leq \boldsymbol{\delta}
$$

where $\mathbf{x}^{k}$ denotes the solution of state variables in the $k$ times of iteration; $\boldsymbol{\delta} \in \mathbf{R}^{11}$ denotes the trust threshold value and is a constant vector.

What follows is the discretization of the linearized state equation, but before discretization, it is imperative to confirm the optimization range and discrete points. We choose the relative distance $r$ as the independent variable of the hypersonic vehicle's penetration problem under investigation in this paper. The work has the following advantages: (1) compared with the separate state of the hypersonic vehicle and the interception missile at a certain moment, we are more concerned about the attack and defense countermeasure in terms of a certain relative distance and (2) if we use time as an independent variable, the appearance of uncertain terminal time $t_{f}$ will make the optimal control problem more complicated. With the relative distance served as the independent variable, the final value of $r$ can be assumed to be the ending point of midcourse guidance of the interception missile. Thus, the terminal penetration angle constraint can also be determined.

Accordingly, $r_{0}$ and $r_{f}$ are chosen as the optimization range of the penetration problem and the problem is discretized by $N+1$ numbers of discrete points whose intervals are equal. Thus, the discrete step length is $\Delta r=\left(r_{f}-r_{0}\right) / N$. Firstly, we discretize the state variable and the control variable on discrete points, namely, $\mathbf{x}_{i}=\mathbf{x}\left(r_{i}\right), \mathbf{u}_{i}=\mathbf{u}\left(r_{i}\right), i=0$, $1, \cdots, N$. Then according to the trapezoidal formula, we perform the mathematical integration of the sequentially 
linearized state equation (namely, equation (9)) with the following formula:

$$
\begin{aligned}
\mathbf{x}_{i}= & \mathbf{x}_{i-1}+\frac{\Delta r}{2}\left[\left(A_{i-1}^{k} \mathbf{x}_{i-1}+B_{i-1}^{k} \mathbf{u}_{i-1}+\mathbf{b}_{i-1}^{k}\right)\right. \\
& \left.+\left(A_{i}^{k} \mathbf{x}_{i}+B_{i}^{k} \mathbf{u}_{i}+\mathbf{b}_{i}^{k}\right)\right],
\end{aligned}
$$

where $A_{i}^{k} \triangleq F_{x}\left(\mathbf{x}^{k}\left(r_{i}\right), \mathbf{u}^{k}\left(r_{i}\right)\right), \quad B_{i}^{k} \triangleq F_{u}\left(\mathbf{x}^{k}\left(r_{i}\right), \mathbf{u}^{k}\left(r_{i}\right)\right)$, and $b_{i}^{k}=b\left(\mathbf{x}^{k}\left(r_{i}\right), \mathbf{u}^{k}\left(r_{i}\right)\right)$.

Equation (12) is further represented as follows:

$$
H_{i-1} \mathbf{x}_{i-1}+H_{i} \mathbf{x}_{i}+G_{i-1} \mathbf{u}_{i-1}+G_{i} \mathbf{u}_{i}=-\frac{\Delta r}{2}\left(\mathbf{b}_{i-1}^{k}+\mathbf{b}_{i}^{k}\right)
$$

where

$$
\begin{aligned}
M & =\left[\begin{array}{cccccccccccc}
I & 0 & 0 & \cdots & 0 & 0 & 0 & 0 & 0 & \cdots & 0 & 0 \\
H_{0} & H_{1} & 0 & \cdots & 0 & 0 & G_{0} & G_{1} & 0 & \cdots & 0 & 0 \\
\vdots & \vdots & \vdots & \ddots & \vdots & \vdots & \vdots & \vdots & \vdots & \ddots & \vdots & \vdots \\
0 & 0 & 0 & \cdots & H_{N-1} & H_{N} & 0 & 0 & 0 & \cdots & G_{N-1} & G_{N}
\end{array}\right], \\
F & =-\frac{\Delta e}{2}\left[\begin{array}{c}
-\left(\frac{2}{\Delta e}\right) \mathbf{x}_{0} \\
\mathbf{b}_{0}^{k}+\mathbf{b}_{1}^{k} \\
\vdots \\
\mathbf{b}_{N-1}^{k}+\mathbf{b}_{N}^{k}
\end{array}\right] .
\end{aligned}
$$

It should be pointed out that the first line of equation in the equality constraints (namely, equation (14)) obtained through linearizing the initial state constraint $\mathbf{x}\left(r_{0}\right)=\mathbf{x}_{0}$.

3.2. Control Constraint Convexification. The original problem $P_{0}$ has control constraints with the angle of attack, bank angle, and mass consumption rate. The constraints on both the angle of attack $a_{T}$ and the mass consumption rate $m_{s}$ are all linear constraints while the constraint on the bank angle is nonlinear and handled in the following ways: the bank angle rate $\dot{\sigma}$ is introduced as a control variable, namely, $\mathbf{u}=\left[\alpha_{T}, \dot{\sigma}, m_{s}\right]^{T} \in \mathbf{R}^{3}$, and the state variable $\sigma$ is also added to the differential function.

3.3. Penetration Angle Constraint Convexification. The interception missile is assumed to use the head-on interception method, and the angles $\left|q_{y}+\theta_{e}\right|$ and $\left|\pi+q_{z}-\psi_{e}\right|$ formed by the missile's line of sight and the hypersonic vehicle's velocity vector are less than 45 degrees. Therefore, we perform the following slackening on formula (4):

$$
\begin{aligned}
\cos \eta & =-\cos q_{y} \cos \theta_{e} \cos \left(q_{z}-\psi_{e}\right)-\sin \theta_{e} \sin q_{y} \\
& <\cos \left(q_{y}+\theta_{e}\right) \cos \left(\pi+q_{z}-\psi_{e}\right) .
\end{aligned}
$$

where $\quad H_{i-1}=I+(\Delta r / 2) A_{i-1}^{k}, \quad H_{i}=-I+(\Delta r / 2) A_{i}^{k}, \quad G_{i-1}=$ $(\Delta r / 2) B_{i-1}^{k}$, and $G_{i}=(\Delta r / 2) B_{i}^{k}$; the number of dimensions of unit matrix $I$ is in agreement with that of coefficient matrix $A$.

All the variables to be optimized are unitarily represented as optimized vector $\mathbf{z}=\left[\begin{array}{llllll}\mathbf{x}_{0}^{T} & \cdots & \mathbf{x}_{N}^{T} & \mathbf{u}_{0}^{T} & \cdots & \mathbf{u}_{N}^{T}\end{array}\right]^{T}$; the linearly discretized state equations are written into the expressions related to $\mathbf{z}$ :

$$
M \mathbf{z}=F,
$$

The nonlinear inequality constraint is then transformed into the concave inequality constraint:

$$
\cos \left(q_{y}+\theta_{e}\right) \cos \left(\pi+q_{z}-\psi_{e}\right)<\cos \eta_{\max }
$$

To show that equation (17) is a concave inequation constraint, we further transform the left hand-side term of the inequation into the following:

$$
\begin{aligned}
& \cos \left(q_{y}+\theta_{e}\right) \cos \left(\pi+q_{z}-\psi_{e}\right) \\
& =\frac{\left(\cos \left(q_{y}+\theta_{e}+q_{z}-\psi_{e}+\pi\right)+\cos \left(q_{y}+\theta_{e}-q_{z}+\psi_{e}-\pi\right)\right)}{2} .
\end{aligned}
$$

According to the abovementioned assumptions $q_{y}+$ $\theta_{e} \in[-\pi / 4, \pi / 4]$ and $\pi+q_{z}-\psi_{e} \in[-\pi / 4, \pi / 4]$, the value ranges of the two cosine functions at the left side of equation (18) are all in $[-\pi / 2, \pi / 2]$; within this scope, the cosine function $\cos x$ is a concave function. Refer to the compound affine mapping theorem and the vector compound theorem [23] in the convex optimization theory. Equation (17) is deduced to be a concave inequation constraint. 
Similar to the differential equation convexification method, the concave inequation constraint can also be approximated with the sequential linearization; the sequential linearization of a concave inequation has already been proved to be able to converge to its optimal solutions [24]. The formula for sequentially linearizing the penetration angle constraint is as follows:

$$
\begin{gathered}
\frac{\partial g}{\partial q_{y}}\left(\mathbf{x}^{k}\right) q_{y}+\frac{\partial g}{\partial q_{z}}\left(\mathbf{x}^{k}\right) q_{z}+\frac{\partial g}{\partial \theta_{e}}\left(\mathbf{x}^{k}\right) \theta_{e} \\
+\frac{\partial g}{\partial \psi_{e}}\left(\mathbf{x}^{k}\right) \psi_{e}<\cos \eta_{\max }+\bar{b},
\end{gathered}
$$

where

$$
\begin{aligned}
& \frac{\partial g}{\partial q_{y}}\left(\mathbf{x}^{k}\right)=\frac{\partial g}{\partial \theta_{e}}\left(\mathbf{x}^{k}\right)=-\sin \left(q_{y}^{k}+\theta_{e}^{k}\right) \cos \left(\pi+q_{z}^{k}-\psi_{e}^{k}\right), \\
& \frac{\partial g}{\partial q_{z}}\left(\mathbf{x}^{k}\right)=-\sin \left(q_{y}^{k}+\theta_{e}^{k}\right) \cos \left(\pi+q_{z}^{k}-\psi_{e}^{k}\right), \\
& \frac{\partial g}{\partial \psi_{e}}\left(\mathbf{x}^{k}\right)=\sin \left(q_{y}^{k}+\theta_{e}^{k}\right) \cos \left(\pi+q_{z}^{k}-\psi_{e}^{k}\right) .
\end{aligned}
$$

$q_{y}^{k}, q_{z}^{k}, \theta_{e}^{k}$, and $\psi_{e}^{k}$ denote the solutions of the $k$ times of iteration; the linearized residual error is as follows:

$$
\begin{aligned}
\bar{b}= & -\cos \left(q_{y}^{k}+\theta_{e}^{k}\right) \cos \left(\pi+q_{z}^{k}-\psi_{e}^{k}\right)+\frac{\partial g}{\partial q_{y}}\left(\mathbf{x}^{k}\right) q_{y}^{k} \\
& +\frac{\partial g}{\partial q_{z}}\left(\mathbf{x}^{k}\right) q_{z}^{k}+\frac{\partial g}{\partial \theta_{e}}\left(\mathbf{x}^{k}\right) \theta_{e}^{k}+\frac{\partial g}{\partial \psi_{e}}\left(\mathbf{x}^{k}\right) \psi_{e}^{k} .
\end{aligned}
$$

Thus, the penetration angle constraint is represented in the form of terminal state constraint qualification, namely,

$$
C\left(\mathbf{x}_{f}^{k}\right) \mathbf{x}_{f}+d_{x}<0
$$

where the coefficient matrix is obtained with sequential linearization equation (19).

3.4. Objective Convexification. Through introducing the intermediate variable $\xi$, the quadratic performance index in problem $P_{0}$ can be equivalently discretized into the following:

$$
\begin{aligned}
\min J & =\sum_{i=1}^{N} \zeta_{i}, \\
\left\|R^{1 / 2} \mathbf{u}_{i}\right\| & \leq \zeta_{i}, \quad i=1, \cdots, N,
\end{aligned}
$$

where $R^{1 / 2}$ is obtained with the square root matrix decomposition formula, namely, $R=\left(R^{1 / 2}\right)^{T} R^{1 / 2}$.

So far, through slackening, sequentially linearizing, and discretizing objective functions, differential equations, and nonlinear inequations, the original penetration problem $P_{0}$ is transformed into a convex problem in its discrete form, noted as problem $P_{1}$ :

$$
\begin{aligned}
& P_{1}: \min J=\sum_{i=1}^{N} \zeta_{i}, \\
& \text { s.t. }\left\{\begin{array}{l}
M \mathbf{z}=F \\
\alpha_{T \min } \leq \alpha_{T \_i} \leq \alpha_{T \max }, \\
\left|\dot{\sigma}_{i}\right| \leq \dot{\sigma}_{\max }, \\
0 \leq m_{s_{-} i} \leq m_{s \max }, \\
C\left(\mathbf{x}_{f}^{k}\right)_{f}+d_{x}<0, \\
\left\|R^{1 / 2} \mathbf{u}_{i}\right\| \leq \zeta_{i}, \\
\left\|\mathbf{x}_{i}-\mathbf{x}_{i}^{k}\right\| \leq \boldsymbol{\delta},
\end{array}\right.
\end{aligned}
$$

where $\|\cdot\|$ is a 2 norm; $i=1,2, \cdots, N$.

Problem $P_{1}$ is a sort of convex problem named secondorder cone problem (SOCP), with a linear objective function and all convex constraints including linear constraints and second-order cone constraints. The optimal solution of the original penetration problem $P_{0}$ can be obtained through iteratively solving problem $P_{1}$. Each iteration process uses solution $\mathbf{x}^{k}$ of the last iteration to update problem $P_{1}$, constantly solves problem $P_{1}$ within a thrust domain, and gradually approximates the optimal solution of problem $P_{0}$ till $\max _{i}\left|\mathbf{x}_{i}^{k+1}-\mathbf{x}_{i}^{k}\right| \leq \boldsymbol{\varepsilon}$. Then the optimization algorithm converges and obtains optimal solutions.

\section{Numerical Demonstrations}

4.1. Settings of Simulation Parameters. The basic parameters and the relevant simulation parameter settings in the attack and defense countermeasure simulation model of the hypersonic vehicle are given in Tables 1 and 2. The boundary values in the control constraints are set as $\alpha_{T \text { min }}=-4^{\circ}$, $\alpha_{T \text { max }}=6^{\circ}, \dot{\sigma}_{\max }=5^{\circ}$, and $m_{s \text { max }}=1$; the boundary value in the penetration angle constraints is set as $\eta_{\max }=20^{\circ}$.

Simulation conditions are set as follows: in the first iteration, control guesses are set to $\mathbf{u}=\left[2^{\circ}, 0,0.2\right]^{T}$, the number of discrete points is set as $N=200$, and the thrust domain constraint values in the formula are set as follows:

$$
\delta=\left[\begin{array}{lllllllllll}
2000 & \frac{20 \pi}{180} & \frac{20 \pi}{180} & 1000 & \frac{20 \pi}{180} & \frac{20 \pi}{180} & 1000 & 1 & \frac{20 \pi}{180} & \frac{20 \pi}{180} & \frac{20 \pi}{180}
\end{array}\right]^{T} .
$$


The error threshold values in the simulation iteration termination condition are set as follows:

$$
\varepsilon=\left[\begin{array}{lllllllllll}
100 & \frac{0.5 \pi}{180} & \frac{0.5 \pi}{180} & 100 & \frac{0.5 \pi}{180} & \frac{0.5 \pi}{180} & 500 & 1 & \frac{0.5 \pi}{180} & \frac{0.5 \pi}{180} & \frac{0.5 \pi}{180}
\end{array}\right]^{T}
$$

The above simulation conditions are used to perform numerical simulation with the YALMIP modeling environment of MATLAB; the primal-dual interior point algorithm in the MOSEK [25] software package is used to solve the SOCP problem. After five times of sequential iteration, the trajectory converges. The time required by each iteration is around $0.4 \mathrm{~s}$; the resolving time required by the interior point algorithm is around $0.2 \mathrm{~s}$. The penetration angle and the hypersonic vehicle's angle of attack calculated by the first four iterations are shown in Figures 3 and 4. The figures show that the sequential convex optimization algorithm converges quickly; the angle-of-attack value solved in the second iteration is already close to the ultimate optimal solution.

In addition, in order to validate the effectiveness of the second-order cone programming (SOCP) method proposed in the paper, numerical results are compared with those obtained by the Gaussian pseudospectral method (GPM). The GPM solves the problem with the sequential quadratic programming (SQP), and we program it by the GPOPS5.1 software package [26]. The GPM has already been proved to obtain its globally optimal solution [27].

The trajectory profile by the SOCP-based method and the GPM (the mesh tolerance is set to $10^{-3}$ ) are shown in Figures 5-7, while the solving times required and optimal performance indexes are given in Table 3. Figure 8 presents the required calculating time of the two methods using different random control initial guesses within control constraints; the horizontal axis only represents the number of initial guesses without any specific physical meaning.

Based on the figures, the SOCP and GPM solutions show very similar trends; the control profiles in Figures 5-7 match well in general. The relatively small differences between the two methods are attributable to the different discretization strategies used in the two solutions, which also occur in reference [28]. On the other hand, the performance of the GPM is only slightly optimal than that of SOCP in terms of the values of 751 and 770, respectively. Therefore, we can at least reckon that the SOCP method is near optimal. Despite the compromise of the optimum objective, the SOCP method performs much better in terms of computational complexity based on Table 3 and Figures 4 and 8. The SOCP-based method in this paper takes an average of 1.3 seconds to generate trajectories, and the peak calculating time for different initial guesses is only 2.45 seconds, while the GPM method takes an average of 7.98 seconds and the longest time can reach 13.1 seconds. To tackle penetration problems, a method with competitive convergence speed and robust solution process is what we always seek for, though it is not rigorously optimal.
Further simulation is conducted to verify whether the penetration angle could function as an index for evaluating the penetration effects when the distance between the missile and the hypersonic vehicle is fixed. In other words, the hypersonic vehicle can successfully escape once it meets certain penetration angle constraints in the attack and defense countermeasure.

Therefore, we design different penetration angle constraints $\left(\eta=10^{\circ}, 15^{\circ}, 20^{\circ}, 25^{\circ}, 30^{\circ}\right)$ and conduct the simulation in which the continuing terminal-course relative motion is considered. In the simulation, when the distance between the attack side and the defense side equals to $r_{f}$, the countermeasure enters the terminal phase where the two sides continue to approach each other till the vehicle is intercepted or successfully escapes. During this period, the guidance law of the interception missile is the pure proportional guidance law with the proportional constant of 3, while the vehicle adopts two kinds of maneuver in order to illustrate the impact of angle constraints. It does not make any maneuver and continues flying at the current speed and direction or maneuvers with maximum overload in the opposite direction of the attacked missile. The maximum overload, about $2.8 \mathrm{~g}$, is obtained by the maximum available attack angle and maximum thrust for a hypersonic vehicle. The formulas for calculating its overload and maneuver direction are as follows:

$$
\begin{aligned}
\overline{\mathbf{n}}_{c \max } & =n_{c \max } \overline{\mathbf{I}}_{n}, \\
n_{c \max } & =\frac{\left(T_{\max } \sin \alpha_{T \max }+L_{T}\left(\alpha_{\mathrm{T} \max }\right)\right)}{m}, \\
\overline{\mathbf{I}}_{n} & =\frac{\left(\bar{V}_{T} \times \overline{\mathrm{Los}}\right) \times \bar{V}_{T}}{\left|\left(\bar{V}_{T} \times \overline{\mathrm{Los}}\right) \times \bar{V}_{T}\right|},
\end{aligned}
$$

where $\overline{\mathbf{I}}_{n}$ represents the maneuver direction; $\bar{V}_{T}$ and $\overline{\text { Los }}$ represent the vehicle's velocity vector and line-of-sight vector, respectively. With various factors being considered, the time constant of an interception missile is set as $0.05 \mathrm{~s}$ and the miss distance is designed as $1 \mathrm{~m}$.

Under different penetration angle constraints and two scenarios of the hypersonic vehicle's continuing motion method, the miss distances of the interception missile in the two scenarios are shown in Table 4. The whole trajectory profiles for the attack side and the defense side are given in Figures 9 and 10, while the figure for comparing the terminal trajectory $\left(20^{\circ}\right.$ penetration angle) in the horizonal plane between different maneuver methods is also shown in Figure 11. 
TABLE 1: The vehicle's basic parameters [8].

\begin{tabular}{lccccc}
\hline Parameters & $C_{L 0}$ & $C_{L}^{\alpha}\left(\mathrm{rad}^{-1}\right)$ & $C_{D 0}$ & $C_{D}^{\alpha^{2}}\left(\mathrm{rad}^{-2}\right)$ & $C_{T}^{m_{s}}\left(\mathrm{~s} \cdot \mathrm{kg}^{-1}\right)$ \\
\hline $\begin{array}{l}\text { Parameter } \\
\text { values }\end{array}$ & -0.013 & 1.833 & 0.015 & 4.596 & 0.162 \\
\hline
\end{tabular}

TABLE 2: The initial values of conditions of the attack side and the defense side.

\begin{tabular}{lc}
\hline Simulation parameters & Parameter values \\
\hline$\left(x_{e 0}, y_{e 0}, z_{e 0}\right)(\mathrm{km})$ & $(100,25,10)$ \\
$V_{e 0}\left(\mathrm{~m} \cdot \mathrm{s}^{-1}\right)$ & 1500 \\
$\left(\theta_{e 0}, \psi_{e 0}\right)(\mathrm{rad})$ & $(0, \pi)$ \\
$\left(x_{i 0}, y_{i 0}, z_{i 0}\right)(\mathrm{km})$ & $(0,0,0)$ \\
$V_{i}\left(\mathrm{~m} \cdot \mathrm{s}^{-1}\right)$ & 1200 \\
$\left(\theta_{i 0}, \psi_{i 0}\right)(\mathrm{rad})$ & $(0.2438,-0.0997)$ \\
$N_{1},\left(N_{2}, N_{3}\right)$ & $5,(0.1,0.1) i / N$ \\
$R$ & $\operatorname{diag}(5,0.1,1)$ \\
$r_{f}(\mathrm{~km})$ & 20 \\
\hline
\end{tabular}

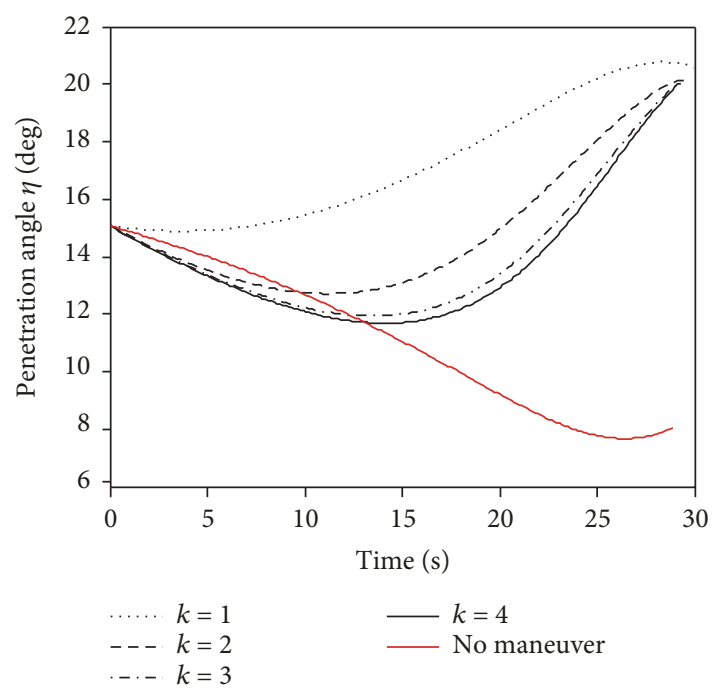

FIGURE 3: Penetration angle in the first four successive iterations of the SOCP-based algorithm.

Table 4 shows that if the penetration angle in the countermeasure is larger than 20 degrees, no matter whether or not the vehicle maneuvers in its terminal countermeasure, it can successfully penetrate. Otherwise, the interception missile will create a head-on strike situation; then even the highoverload terminal maneuver of the hypersonic vehicle cannot evade the attack by an interception missile. The penetration angle successfully transforms the complicated countermeasure process of the hypersonic vehicle's evasion into a simple penetration angle constraint. The penetration can be accomplished without any energy waste.

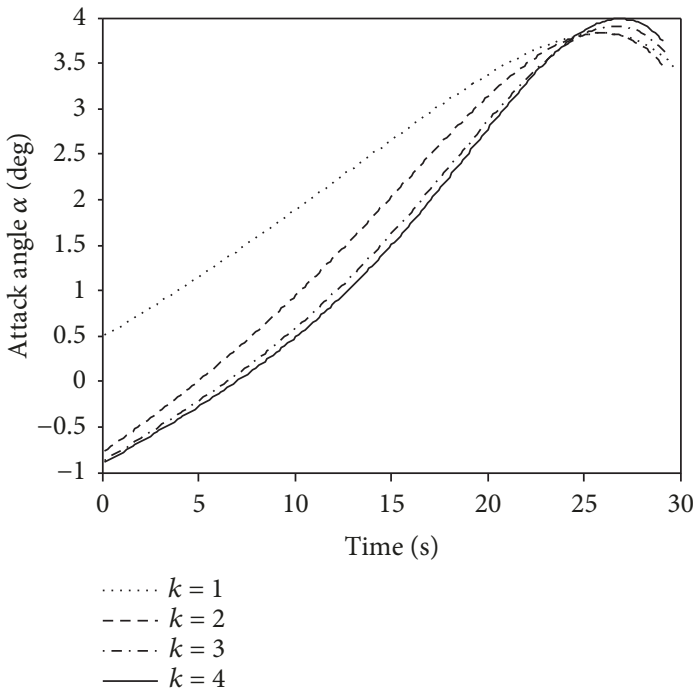

FIgURe 4: Angle-of-attack profile in the first four successive iterations of the SOCP-based algorithm.

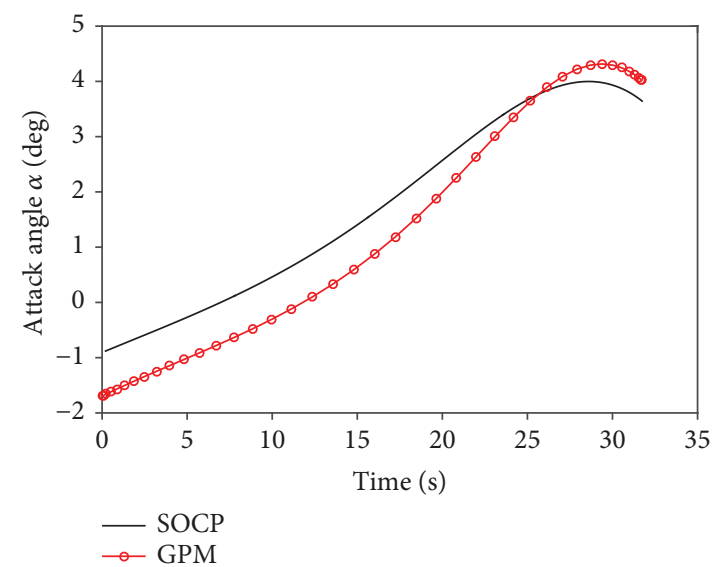

FIgURE 5: The penetrator's attack angle profiles, using the two methods.

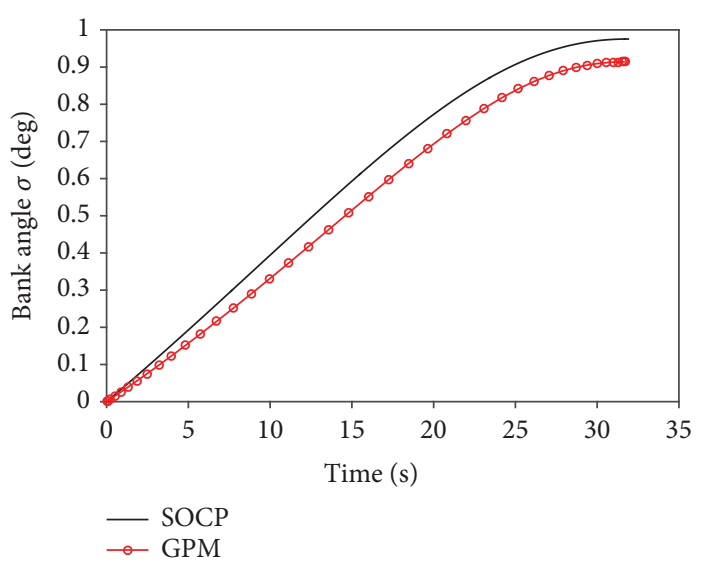

FIgURE 6: The penetrator's banking angle profiles, using the two methods. 


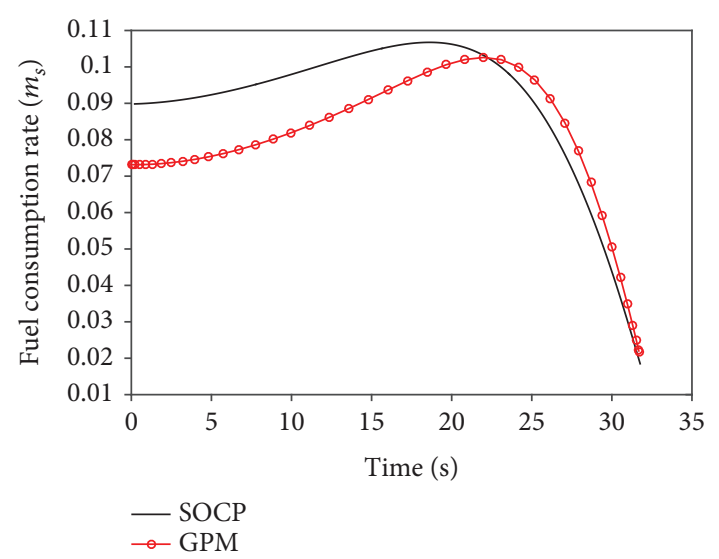

FIGURE 7: The penetrator's fuel consumption rate profiles, using the two methods.

TABle 3: Comparison of the time consumed with performance indexes, using the SOCP and GPM methods.

\begin{tabular}{lcccc}
\hline Method & Discrete point & Iteration & Time (s) & Performance index \\
\hline GPM & 40 & - & 7.76 & 751 \\
SOCP & 200 & 5 & 1.42 & 770 \\
\hline
\end{tabular}

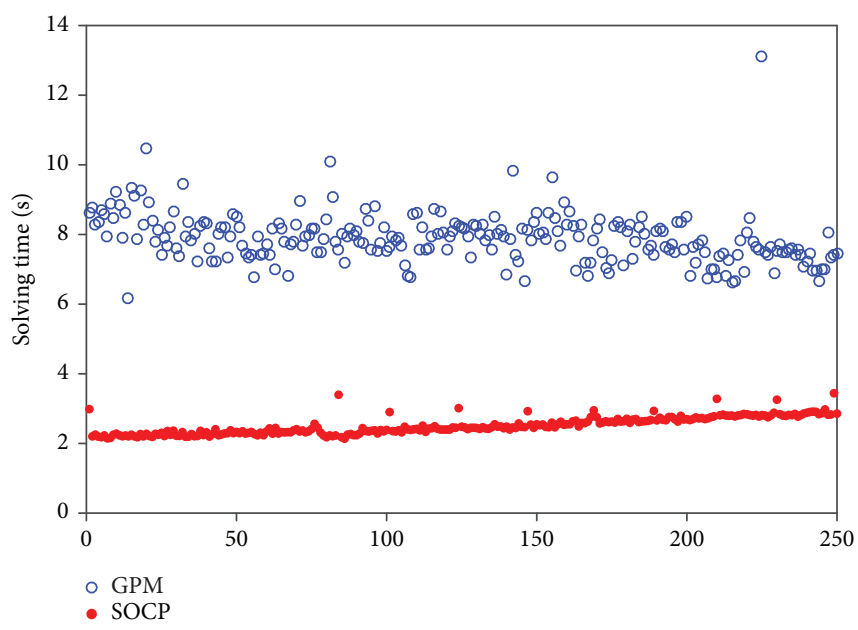

FIGURE 8: Calculating time for different initial guesses.

TABle 4: Miss distances in the two scenarios of the terminal maximum overload maneuver or no maneuver.

\begin{tabular}{lcc}
\hline Penetration angle & $\begin{array}{c}\text { Miss distance } \\
\text { (no maneuver) }\end{array}$ & $\begin{array}{c}\text { Miss distance } \\
\text { (maneuver) }\end{array}$ \\
\hline 10 & 0.15 & 0.26 \\
15 & 0.30 & 0.78 \\
20 & 2.89 & 74.36 \\
25 & 253.25 & 757.9 \\
30 & 1705.34 & 1827.27 \\
\hline
\end{tabular}

\section{Conclusions}

Confronted with the serious interception threat, hypersonic vehicles need to be empowered with the ability to generate

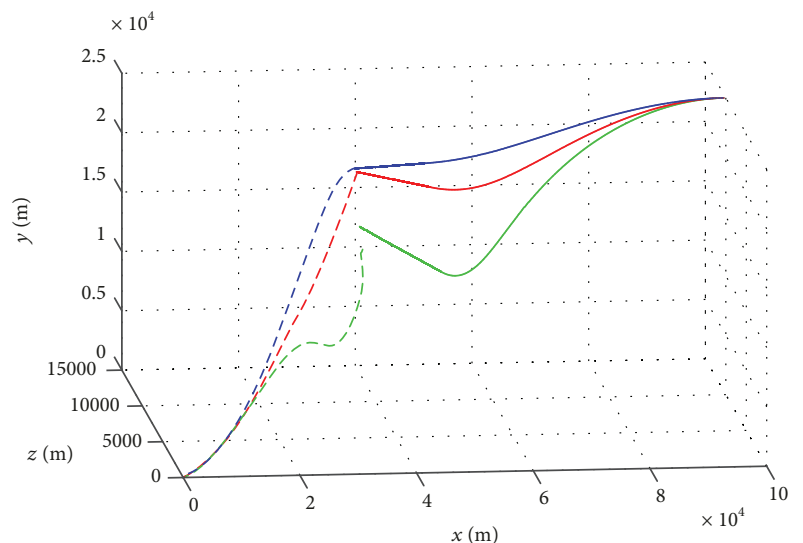

— Penetration trajectory $(\eta=10) \quad--$ - Interception trajectory $(\eta=20)$ -- Interception trajectory $(\eta=10)-$ Penetration trajectory $(\eta=30)$
Penetration trajectory $(\eta=20)--$ Interception trajectory $(\eta=30)$

FIgURE 9: The attack and defense trajectories in the scenario of no terminal maneuver.

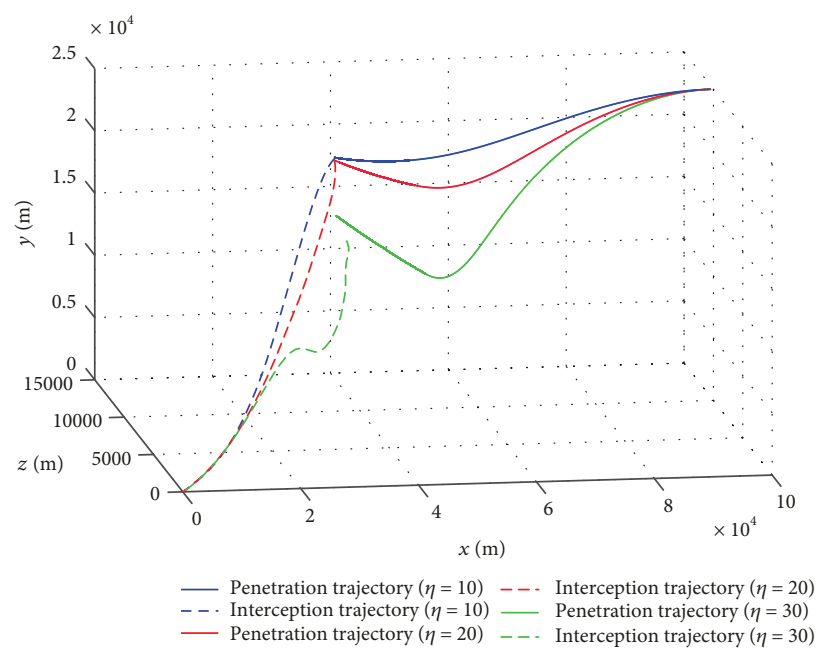

FIgURE 10: The attack and defense trajectories in the scenario of the maximum overload terminal maneuver.

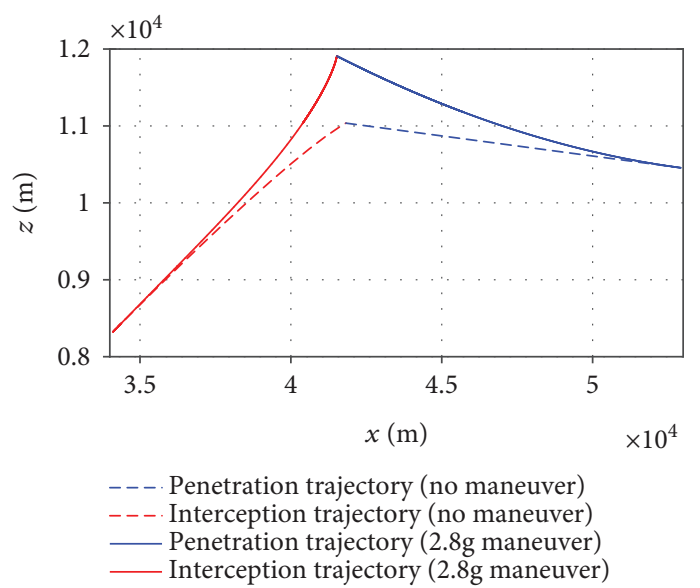

FIGURE 11: The attack and defense trajectories in the scenarios of no maneuver and maximum overload maneuver (horizonal plane). 
a penetration trajectory, cope with air defense missiles, and attack targets simultaneously. In this paper, a rapid penetration trajectory programming method is proposed to guide the hypersonic vehicle to evade against a head-on interception missile with the minimum energy consumption. It has been demonstrated that the method raised in this paper is effective and has competitive computational performances including a quick convergence rate, rapid calculation speed, and robustness of initial guesses. Moreover, the penetration angle summarized in this paper proves to be a valid index of the penetration effect, facilitating the application of optimization methods to hypersonic penetration problems. As a whole, these research results contribute to the future online application of hypersonic penetration programming.

\section{Data Availability}

The data used to support the findings of this study are included within the article.

\section{Conflicts of Interest}

The authors declare that they have no conflicts of interest.

\section{Acknowledgments}

The research involving this paper is supported by the NSFA (Grant no: U1730135).

\section{References}

[1] R. Kazmar, Airbreathing hypersonic propulsion at Pratt \& Whitney - overview, Pratt and Whitney, West Palm Beach, 2006.

[2] M. A. Bolender and D. B. Doman, "Nonlinear longitudinal dynamical model of an air-breathing hypersonic vehicle," Journal of Spacecraft and Rockets, vol. 44, no. 2, pp. 374-387, 2007.

[3] S. Keshmiri, M. Mirmirani, and R. Colgren, "Six-DOF modeling and simulation of a generic hypersonic vehicle for conceptual design studies," in AIAA Modeling and Simulation Technologies Conference and Exhibit, Providence, Rhode Island, 2004.

[4] V. Shaferman and T. Shima, "Linear quadratic differential games guidance law for imposing a terminal intercept angle," in AIAA Guidance, Navigation and Control Conference and Exhibit, Honolulu, Hawaii, 2008.

[5] M. H. Hsueh, C. I. Huang, and L. C. Fu, "A differential game based guidance law for the interceptor missiles," in IECON 2007 - 33rd Annual Conference of the IEEE Industrial Electronics Society, pp. 665-670, Taipei, Taiwan, 2007.

[6] R. Bardhan and D. Ghose, "Nonlinear differential games-based impact-angle-constrained guidance law," Journal of Guidance, Control, and Dynamics, vol. 38, no. 3, pp. 384-402, 2015.

[7] P. N. Dwivedi, A. Bhattacharya, and R. Padhi, "Suboptimal midcourse guidance of interceptors for high-speed targets with alignment angle constraint," Journal of Guidance, Control, and Dynamics, vol. 34, no. 3, pp. 860-877, 2011.

[8] H. Gou, W.-x. Fu, B. Fu, K. Chen, and J. Yan, "Penetration trajectory programming for air-breathing hypersonic vehicles in cruise duration with control constraints and flight dynamics uncertainties," in AIAA International Space Planes and Hypersonics Technologies Conference, Xiamen, China, 2017.
[9] S. M. Roberts, Two-Point Boundary Value Problems: Shooting Methods, American Elsevier Publishing Company, 1972.

[10] L. D. Berkovitz, Optimal Control Theory, Springer-Verlag, 1974.

[11] T. Jorris, C. Schulz, F. Friedl, and A. Rao, "Constrained Trajectory Optimization Using Pseudospectral Methods," in AIAA Atmospheric Flight Mechanics Conference and Exhibit, Honolulu, Hawaii, 2013.

[12] C. L. Darby, W. W. Hager, and A. V. Rao, "An hp-adaptive pseudospectral method for solving optimal control problems," Optimal Control Applications \& Methods, vol. 32, no. 4, pp. 476-502, 2011.

[13] A. Joos, C. Seiferth, L. Schmitt, and W. Fichter, "Parameters for nonlinear model predictive control in unmanned aerial vehicle path-planning applications," Journal of Guidance, Control, and Dynamics, vol. 40, no. 2, pp. 484-492, 2017.

[14] R. Padhi and M. Kothari, "Model predictive static programming: a computationally efficient technique for suboptimal control design," International Journal of Innovative Computing, Information and Control, vol. 5, no. 2, pp. 399-411, 2009.

[15] B. Acikmese, D. Scharf, L. Blackmore, and A. Wolf, "Enhancements on the convex programming based powered descent guidance algorithm for Mars landing," in AIAA/AAS Astrodynamics Specialist Conference and Exhibit, Honolulu, Hawaii, 2008.

[16] B. Acikmese and S. R. Ploen, "Convex programming approach to powered descent guidance for Mars landing," Journal of Guidance, Control, and Dynamics, vol. 30, no. 5, pp. 13531366, 2007.

[17] P. Zarchan, Tactical and strategic missile guidance, American Institute of Aeronautics \& Astronautics Inc, 6 edition, 1994.

[18] N. Indig, J. Z. Benasher, and N. Farber, "Near-optimal spatial midcourse guidance law with an angular constraint," Journal of Guidance, Control, and Dynamics, vol. 37, no. 1, pp. 214223, 2014.

[19] W. Jingchuan, Y. Baoqing, and W. Kaining, "Target allocation method based on intercept geometry," in 2015 34th Chinese Control Conference (CCC), Hangzhou, China, July 2015.

[20] S. Lei, H. Kexi, C. Xiaofei, and J. Yan, "Researching interception of near-space hypersonic cruise missile," Journal of Northwestern Polytechnical University, vol. 33, no. 4, pp. 615-620, 2015.

[21] L. Yang, Z. Jiayi, and L. Shumin, "Review and prospect of nonlinear control theory," Journal of Winged Missiles, vol. 11, no. 11, pp. 55-58, 2004.

[22] M. Tomás-Rodríguez and S. P. Banks, Linear, Time-Varying Approximations to Nonlinear Dynamical Systems, Springer, 2010.

[23] S. Boyd and L. Vandenberghe, Convex Optimization, World Book Inc., 2013.

[24] X. Liu and P. Lu, "Solving nonconvex optimal control problems by convex optimization," Journal of Guidance, Control, and Dynamics, vol. 37, no. 3, pp. 750-765, 2014.

[25] E. D. Andersen and K. D. Andersen, "The Mosek Interior Point Optimizer for Linear Programming: An Implementation of the Homogeneous Algorithm," in High Performance Optimization, pp. 197-232, Springer, 2000.

[26] M. A. Patterson and A. V. Rao, GPOPS-II Version 1.0: A General-Purpose MATLAB Toolbox for Solving Optimal Control Problems Using the Radau Pseudospectral Method, University of Florida, 2013. 
[27] B. D. A. Gauss, Pseudospectral transcription for optimal control, Massachusetts Institute of Technology, Colorado, 2005.

[28] X. Liu, Z. Shen, and P. Lu, "Entry trajectory optimization by second-order cone programming," Journal of Guidance, Control, and Dynamics, vol. 39, no. 2, pp. 227-241, 2015. 


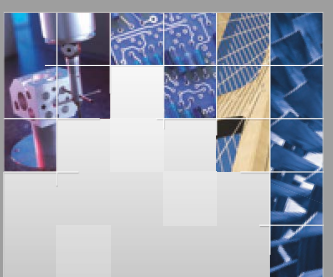

\section{Enfincering}
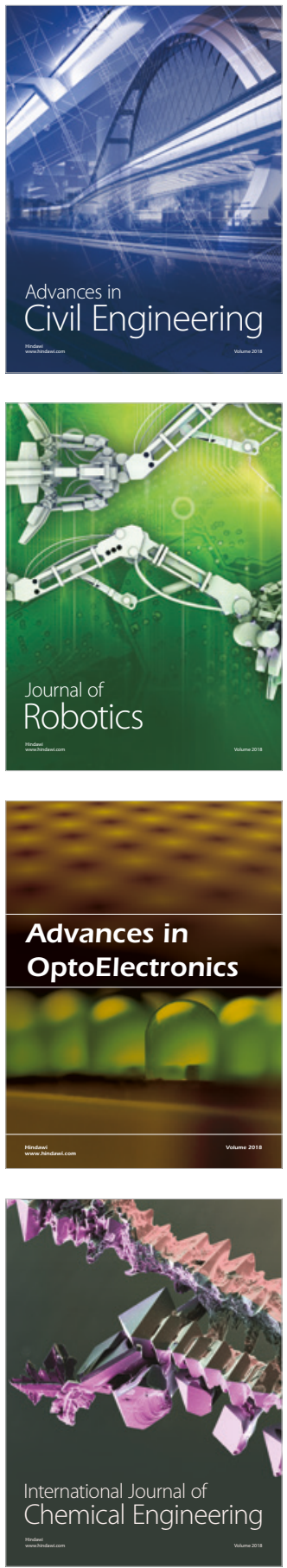

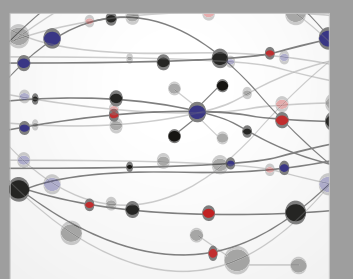

\section{Rotating \\ Machinery}

The Scientific World Journal

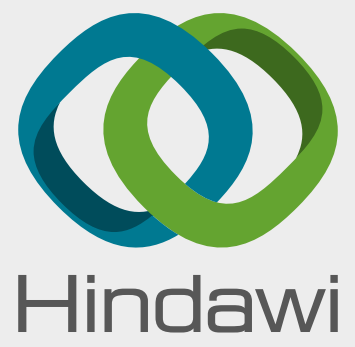

Submit your manuscripts at

www.hindawi.com
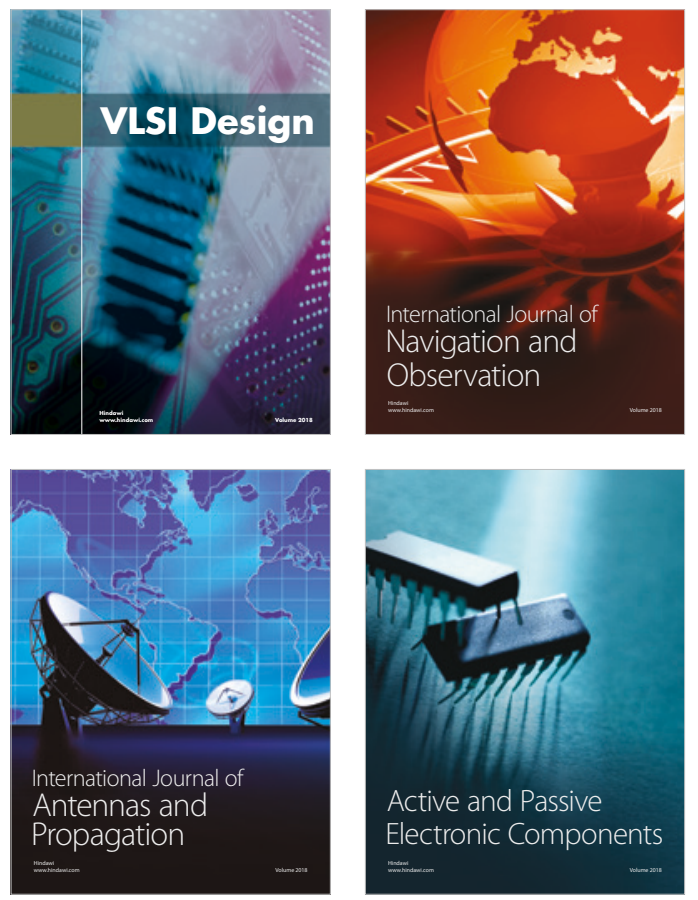
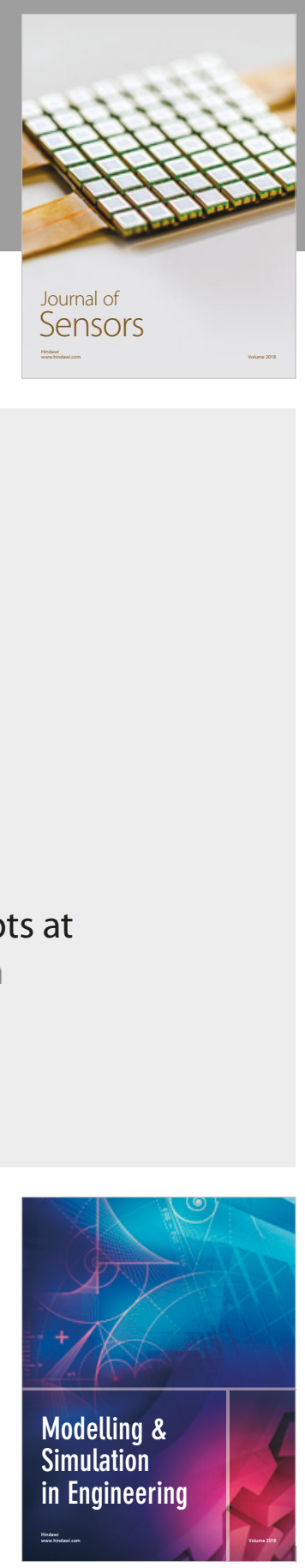

\section{Advances \\ Multimedia}
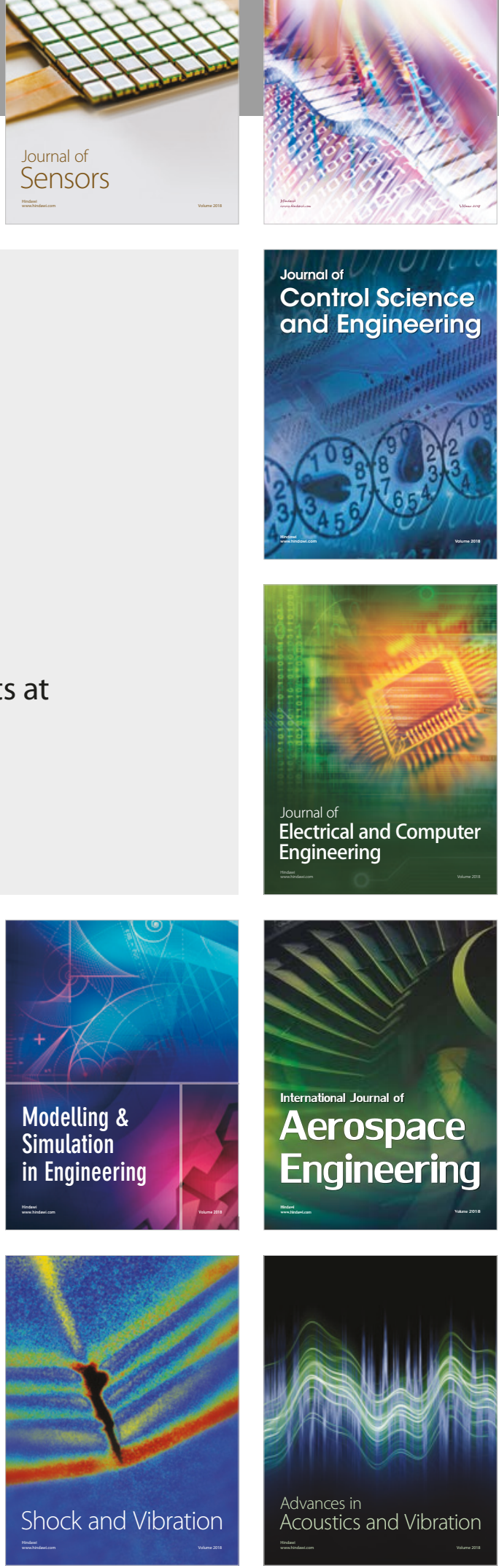